\title{
Author Index to Volume 37
}

Abdel-Mageed, A, 806

Ács, Z, 714

Akselrod, S, 294

Albuquerque, MLC, 10

Alkaddour, HS, 820

Amato, M, 145

Anderson, GG, 586

Andrew, M, 373

Arsan, S, 233

Asakura, H, 303

Aunis, D, 101

Avison, MJ, 244

Avner, ED, 755

Ayas, M, 202

Aynsley-Green, A, 409

Baarsma, R, 626

Babbot, CC, 56

Baertschi, AJ, 310

Baggiani, AM, 571

Bailey, RC, 507

Ball, KT, 303

Bancalari, E, 617

Barbarino-Monnier, P, 101

Barbé, F, 101

Bard, H, 361

Barna, I, 714

Barsoum-Homsy, M, 140

Bartlett, K, 354

Battaglia, FC, 571, 764

Beauloye, V, 334

Becker, L, 165

Bedell, K, 741

Bedford Russell, AR, 630

Belik, J, 196

Belmonte, DC, 310

Benoit, J, 140

Benson, DW, Jr., 289

Berry, L, 373

Berry, SM, 450

Bersch, N, 507

Bitar, KN, 202

Blain-NeIson, PL, 75

Blanchette-Mackie, EJ, 687

Bland, RD, 265

Blennow, M, 260

Blot, P, 64

Boesch, C, 145

Boksa, P, 489

Bossi, E, 145

Bouquet, J, 213

Bourdony, CJ, 820

Boutroy, MJ, 101

Brackett, JC, 675

Brady, RO, 687

Brans, YW, 94

Breuss, J, 561

Briggs, K, 764

Brooks, JO, III, 460

Brooks, W, 489

Brown, CL, 21
Brown, GC, 667

Brown, T, 165

Bruin, NC, 94

Bryant, JL, 56

Buchanan, G, Jr., 806

Bugovics, G, 714

Bullock, RE, 409

Burri, PH, 783, 789

Burri, R, 145

Burrin, DG, 593

Buscaglia, M, 571

Cady, EB, 667

Campbell, KP, 693

Canani, RB, 576

Carbone, GMR, 316

Carlson, SE, 581

Carlton, DP, 265

Carnielli, VP, 381

Carpenter, TO, 726

Casola, A, 576

Cassin, S, 35

Casteels, M, 424

Cetin, I, 571

Chang, YT, 820

Chatow, U, 294

Chemtob, S, 81

Chen, C-M, 271

Chen, F, 277

Chen, Y, 469, 611

Cherry, JD, 623

Chesney, RW, 227

Chevalier, RL, 310

Chheda, S, 444

Chiumello, G, 736

Cho, SC, 265

Christensen, RD, 806

Christian, J, 365

Cingari, DS, 777

Cinquanta, L, 106

Clark, EB, 465

Claure, N, 617

Clayton, PT, 424

Clements, JA, 21

Clyman, RI, 561

Colombini, J, 736

Comi, G, 736

Cooper, BG, 409

Cooper, CE, 667

Cortez, AB, 507

Cosyns, M, 455

Cripps, AW, 155

Czernichow, P, 731

Dalle, D, 482

Danko, I, 693

David, ES, 777

David-Cu, R, 26

Davidson, S, 294

Davis, P, 265

Davis, S, 707

Davis, TA, 593 de Bont, ESJM, 626

Degenhart, HJ, 94, 381

De Groote, D, 64

DeJonge, $\mathrm{MH}, 418$

de Leij, LHFM, 626

Delivoria-Papadopoulos, M, 133

Delorme, M, 373

DeMarco, VG, 35

de Moor, $\mathrm{CH}, 681$

Deng, L, 450

Dhondt, J-L, 151

Diaham, B, 606

DiFiore, JM, 796

Dolan, LM, 820

Dollberg, S, 31

Donaldson, DL, 820

Dressendörfer, RA, 502

Duara, S, 617

Dwyer, NK, 687

Eagles, J, 389

Ebner, S, 593

Edrington, JL, 10

Edwards, $\mathrm{AD}, 667$

Eidson, TH, 10

Eljaafari, A, 64

Ellis, B, 726

Eriksson, U, 343

Escobar, O, 321

Eyre, JA, 409

Fairweather-Tait, S, 389

Federico, G, 106

Fennessey, PV, 571, 764

Ferrante, A, 155

Ferraris, RP, 777

Findlay, RD, 26

Finegold, DN, 675

Fink, C, 165

Finkelstein, JN, 189

Fiorotto, ML, 94, 593

Fowden, AL, 207

Fox, T, 389

Franchimont, P, 64

Frank, L, 469, 611

Franks, RR, 56

Frates, RC, Jr., 460

Frenkel, LM, 623

Fritz, JD, 693

Fujita, I, 646

Fukushima, K, 160

Fusch, C, 145

Gac, SL, 64

Gagnon, C, 361

Garakian, A, 623

Garibaldi, LR, 820

Garmey, MJ, 310

Garofalo, R, 444

Geffner, ME, 507

George, EK, 213

Gerhardt, TO, 617
Gevers, M, 800

Gibson, FM, 630

Gijsbers, CFM, 213

Gil, A, 328

Giroir, BP, 165

Glazier, JD, 720

Gleason, CA, 661

Gluckman, PD, 303, 707

Goh, DHB, 155

Golde, DW, 507

Goldin, E, 687

Goldman, AS, 444

Gordon-Smith, EC, 630

Graham Davies, E, 630

Guarino, A, 576

Guilhot, F, 151

Guillozo, H, 606

Gunn, TR, 303

Gunning, MI, 707

Hack, WWM, 800

Hagberg, H, 260

Halliday, D, 381

Hammarlund, K, 233

Hammond, KB, 460

Hanna, N, 81

Hansen, AL, 465

Hara, T, 437

Hawgood, S, 21

Hay, WW, Jr., 365

Hayward, AR, 455

Heikamp, A, 476

Heinrich, U, 86

Hempe, JM, 321

Henwick, S, 70

Herschkowitz, N, 145, 244

Hetherington, SV, 70

Hillemeier, C, 202

Hoath, SB, 402

Hoffman, EP, 675

Hoffmann, GF, 1

Hoganson, GE, Jr., 432

Hökfelt, T, 687

Holgert, H, 687

Holmberg, C, 640

Holtzman, D, 253

Honig, GR, 432

Hoop, RC, 675

Hopman, JCW, 124

Horowitz, S, 189

Horswill, CA, 600

Houwen, RHJ, 213

$\mathrm{Hu}, \mathrm{N}, 465$

Hughes, SF, 289

Hüppi, PS, 145

Iafusco, M, 576

Ichijo, M, 437

Ikegami, M, 271

Ingrand, P, 151

Irie, K, 437

Ishiguro, A, 160 
Jackson, S, 354

Jacobson, LJ, 365

Jaeken, J, 395

Jakab, M, 175

Jansen, M, 681

Jarvis, JN, 450

Jobe, AH, 271

Johnston, BM, 707

Jones, DP, 227

Jørgensen, PE, 169, 175

Kajio, F, 117

Kalenga, M, 783, 789

Kalimo, H, 634

Káposzta, R, 50

Kärkkäinen, J, 640

Keller, BB, 465

Kelley, RI, 671

Kelly, DP, 581

Kessler, U, 502

Ketelslegers, J-M, 334

Kianicka, I, 482

Kien, CL, 600

Kiess, W, 502

Kikkawa, Y, 646

Kimpen, JLL, 626

Kinter, MT, 41

Kirkbride, V, 667

Kjartansson, S, 233

Klitzner, TS, 277

Klotman, PE, 56

Koehler, RC, 661

Koenig, JI, 714

Koldovsky, O, 586

Kollée, LAA, 124

König, A, 502

Koo, WWK, 581

Kramer, RH, 561

Kratz, L, 671

Krishnamurthy, A, 489

Krogerus, L, 640

Krugman, SD, 365

Lachapelle, P, 81

Lafeber, HN, 800

Lagercrantz, H, 15, 687

Laine, J, 640

Lake, M, 586

Lane, $\mathrm{PH}, 747$

Larsson, H, 652

Latendresse, JS, 693

Lavandosky, G, 165

Lawson, AM, 424

Leffler, CW, 10

Leger, J, 731

Leite, N, 606

Lemonnier, D, 606

Lendahl, U, 15

Leroux, J-F, 482

$\mathrm{Li}, \mathrm{Y}, 806$

Lia, C, 736

Lin, J, 652

Lin, K, 820

Lindberg, T, 771

Ling, $X, 373$

Locatelli, T, 736

LoMonaco, MB, 189

Lont, M, 265

Lorek, A, 667

Mack, DR, 75
Maiter, D, 334

Majander, A, 634

Mäkelä-Bengs, P, 634

Maldonado, J, 328

Mallard, EC, 707

Manco-Johnson, MJ, 365

Mandell, L, 806

Marconi, AM, 571

Marks, J, 165

Maródi, L, 50

Marro, PJ, 133

Mason, JI, 820

Matthews, DSF, 409

Matthews, JNS, 409

Mauray, F, 561

Mawer, EB, 720

Mayatepek, E, 1

Mazingue, F, 151

Mazursky, JE, 741

McAvoy, S, 593

McCoy, KS, 600

McCracken, DM, 586

McGowan, JE, 133

Mearin, ML, 213

Mechinaud, F, 151

Medaglini, S, 736

Mehls, O, 86

Mei, F, 444

Meidert, A, 502

Mieli-Vergani, G, 424

Miki, M, 219

Milerad, J, 652

Miller, LA, 227

Miller, MJ, 796

Millot, F, 151

Mino, M, 219

Mishra, OP, 133

Mitchell, L, 373

Miyazaki, S, 437

Mizuno, K, 271

Moftaquir-Handaj, A, 101

Moore, $\mathrm{H}, 450$

Mora, S, 736

Morishima, M, 117

Muaku, SM, 334

Muchant, DG, 310

Myatt, L, 31

Nakazawa, M, 117

Naruse, H, 253

Nauta, J, 755

Naylor, EW, 675

Ndiaye, B, 606

Nexø, E, 169, 175

Nielsen, K, 623

Ning, $\mathrm{X}-\mathrm{H}, 182$

Noel, M, 731

Norén, O, 207

Notkins, AL, 56

Novotny, EJ, Jr., 244

Nuutila, A, 634

Nuyt, A-M, 741

Nybacka, O, 343

O’Dorisio, TM, 600

Oetomo, SB, 476

Ofosu, F, 373

Ogihara, H, 219

Ogihara, T, 219

Ohls, RK, 806

Okah, FA, 402
Okken, A, 626

Orquin, J, 81, 140

Paes, B, 373

Page, WV, 741

Paillard, F, 64

Palkowetz, KH, 444

Pang, S, 820

Pardi, G, 571

Patrick, CC, 70

Pence, RA, 310

Penrice, J, 667

Pentchev, PG, 687

Pequignot, J-M, 687

Peri, KG, 361

Persson, H, 15

Peters, M, 365

Petroff, OAC, 244

Phelan, CM, 289

Philipps, AF, 586

Pickens, WL, 402

Pihko, H, 634

Pirenne-Ansart, $\mathrm{H}, 64$

Plötz, FB, 476

Portman, MA, 182

Postel-Vinay, M-C, 731

Poulsen, SS, 169, 175

Pourfarzam, M, 354

Power, GG, 303

Praud, J-P, 482

Price, LT, 469

Prichard, JW, 244

Prina Cerai, LM, 736

Prudhon, C, 606

Pytela, R, 561

Qin, W, 283

Raaberg, L, 169, 175

Rajaram, S, 581

Ramírez, M, 328

Rangachari, A, 581

Rao, R, 586

Rapola, J, 634

Rassin, DK, 444

Ray, PE, 56

Reeds, PJ, 593

Reichman, BL, 294

Reynolds, EOR, 667

Richardson, CJ, 460

Rigler, O, 497

Rijkers, GT, 812

Ringstedt, T, 15

Roberds, SL, 693

Roberson, PK, 70

Roberts, DD, 460

Roberts, RJ, 41

Robertson, DM, 155

Robillard, JE, 741

Romero, R, 450

Rondanini, GF, 736

Rose, JC, 316

Rose, VC, 661

Rosengren, L, 260

Rosseneu, M, 328

Rothman, DL, 244

Roy, M-S, 81, 140

Rozin, A, 497

Rubino, A, 576

Rudloff, HE, 444

Russo, R, 576
Rutledge, JC, 755

Sack, J, 497

Saggese, G, 106

Saito, S, 437

Sall, MG, 606

Salo, MK, 640

Sánchez-Pozo, A, 328

Sanders, EAM, 812

Sanders, RS, 189

Sandoval, M, 321

Sangild, PT, 207

Santoro, TJ, 56

Sauer, PJJ, 381

Schaefer, J, 354

Schlueter, MA, 21

Schmalstieg, FC, 444

Schneider, JA, 283

Schoknecht, PA, 593

Schriever, K, 502

Schultz, JC, 432

Schwarz, HP, 502

Scott, S, 460

Scott, SM, 112

Seashore, MR, 244

Sedin, G, 233

Segar, JL, 741

Serenius, F, 771

Shaffner, DH, 661

Shahidi, NT, 432

Shannon, KM, 277

Sheikh, AU, 316

Shimbo, T, 160

Sibley, CP, 720

Silva Neto, G, 617

Silver, M, 207

Sindhu, RK, 646

Sinkin, RA, 189

Siragusa, V, 736

Sjörs, G, 233

Sjöström, H, 207

Skimming, JW, 35

Sloas, MM, 70

Sosenko, IRS, 469, 611

Sparks, JW, 365

Spedale, S, 365

Spitz, DR, 41

Sterkers, G, 64

Stevens, H, 476

Stoelinga, GBA, 124

Strasburger, CJ, 502

Strauss, AW, 675

Styrud, J, 343

Sulkers, EJ, 381

Sundell, HW, 652

Suomalainen, A, 634

Sussenbach, JS, 681

Sweeney, WE, 755

Taeusch, HW, 26

Tan, A-M, 155

Tang, L-Q, 15

Tannenbaum, JE, 561

Tenbergen-Meekes, A-M, 812

Thissen, J-P, 334

Thoresen, M, 667

Thunberg, L, 343

Thureen, PJ, 764

Tomita, H, 117

Tomita, SM, 117

Tönshoff, B, 86 
Törnhage, C-J, 771

Townsend, SF, 365

Traystman, RJ, 661

Tschanz, SA, 783, 789

Tsuji, M, 253

Turnbull, DM, 354

Ueda, T, 271

Underwood, LE, 334

Uvnäs-Moberg, K, 771

Van Biervliet, JP, 328

Van den Brande, JL, 681

Vandenbroucke, JP, 213

van der Mooren, K, 800

van der Velde, EA, 213

Van Dop, C, 277

van Geijn, HP, 124 van Genderingen, HR, 800

Van Goudoever, JB, 381

van Ravenswaaij-Arts, CMA, 124

Vaquero, C, 64

Vargas, A, 321

Varma, DR, 81

Veldhuis, JD, 86

Vida, LN, 432

Visser, HKA, 94

Volpe, J, 253

Voorhorst-Ogink, MM, 812

Wade, S, 606

Wahlroos, O, 640

Waleh, NS, 561

Walker, MW, 41
Walther, FJ, 26

Warner, BW, 31

Watterberg, KL, 112

Wattimena, JLD, 381

Weber, G, 736

Weller, A, 497

Westerhof, N, 800

Wharf, SG, 389

Whitehead, RG, 239

Widness, JA, 361

Williams, CE, 707

Wolff, JA, 693

Woods, CG, 283

Woods, WT, Jr., 283

Wyatt, JS, 667

Wylezinska, M, 667
Yamada, M, 437

Yanai, N, 437

Yang, $\mathrm{X}, 820$

Yasuda, H, 219

Yukitake, K, 21

Zachman, RD, 418

Zegers, BJM, 812

Zegher, F, 395

Zeghoud, F, 606

Zehnder, T, 316

Zhang, L, 820

Zhang, Y, 455

Ziadeh, R, 675

Ziegler, EE, 361

Zipf, WB, 600

Zuckerman, SL, 10 